\title{
Consensus formation on coevolving networks: groups' formation and structure
}

\author{
Balazs Kozma ${ }^{1}$ and Alain Barrat ${ }^{1,2}$ \\ ${ }^{1}$ LPT, CNRS, UMR 8627, and Univ Paris-Sud, Orsay, F-91405, France \\ ${ }^{2}$ Complex Networks Lagrange Laboratory, ISI Foundation, Turin, Italy \\ E-mail: kozmab@th.u-psud.fr, alain.barrat@u-psud.fr
}

\begin{abstract}
.
We study the effect of adaptivity on a social model of opinion dynamics and consensus formation. We analyze how the adaptivity of the network of contacts between agents to the underlying social dynamics affects the size and topological properties of groups and the convergence time to the stable final state. We find that, while on static networks these properties are determined by percolation phenomena, on adaptive networks the rewiring process leads to different behaviors: Adaptive rewiring fosters group formation by enhancing communication between agents of similar opinion, though it also makes possible the division of clusters. We show how the convergence time is determined by the characteristic time of link rearrangement. We finally investigate how the adaptivity yields nontrivial correlations between the internal topology and the size of the groups of agreeing agents.

PACS numbers: 89.75.-k, -87.23.Ge, 05.40.-a
\end{abstract}

Submitted to: J. Phys. A: Math. Gen.

\section{Introduction}

In the last years, many efforts have been devoted to the understanding of how the behavior of models of interacting agents is affected by the topology of these interactions. Various simple models have indeed been defined in order to study how local rules of evolution can lead to the emergence of global phenomena. This topic, at the center of the statistical physics, has been connected to the field of social sciences, leading to the so-called sociophysics [1]. The statistical physics approach starts from the simplest possible models, in which each agent is defined by an internal state, which evolves according to certain rules of interactions with the neighboring agents. The internal state can be an Ising variable taking only two possible values $[2,3,4]$, a vector of traits [5], a continuous variable $[6,7,8,9,10]$, or have a more complex structure $[11,12,13]$. The local interaction rules may involve the simple imitation of a neighbor, an alignment to a local majority, or more involved negotiation processes. The main question concerns 
in all cases the possibility of the appearance of a global consensus, defined by the fact that all agents' internal states coincide, without the need for any central authority to supervise such behavior. Alternatively, polarized states in which several coexisting states or opinions survive can be obtained, and the parameters of the model drive the transition between consensus and polarization.

The science of complex networks has recently led to an intense activity devoted to the characterization and the understanding of the topology of man-made and natural networks $[14,15,16,17,18,19,20,21]$, such as social networks, which determine how social agents interact. Numerous studies have revealed the ubiquity of various striking characteristics, such as the small-world effect: although each node has a number of neighbors which remains small with respect to the total number of agents, only a small number of hops suffices to go from any agent to any other on the network. This has prompted the investigation of the effect of various interaction topologies on the behavior of agents connected according to these topologies, highlighting the relevance of smallworld and heterogeneous structures (see for example [22, 23, 24, 25]).

More recently, the focus has shifted to take into account the fact that many networks, and in particular social networks, are dynamical in nature: links appear and disappear continuously in time, on many timescales. Moreover, such modifications of the network's topology do not occur independently from the agents' states but as a feedback effect: the topology determines the evolution of the agents' opinions, which in its turn determines how the topology can be modified [26, 27, 28, 29, 30, 31, 32]; the network becomes adaptive. In this framework, we consider here the coevolution of an adaptive network of interacting agents, and investigate how the final state of the system depends on this coevolution. We focus on the Deffuant model in which opinions are continuous variables and neighboring agents which have close enough opinions (as determined by a tolerance parameter) can reach a local consensus. In our model, the rate of evolution of the network's topology is tunable and represents one of the parameters. We focus on simple evolution rules of the topology, that do not require prior knowledge of the state of agents to which new links are established. We study the role of the various parameters such as the tolerance of agents and the rate of topology evolution. As already shown in a previous investigation [32], the possibility of the interaction network to adapt to the changes in the opinion of the agents has important consequences on the evolution mechanisms and on the structure of the system's final state. We focus here on the one hand on the convergence time needed to reach this final state, and on the other hand on the detailed characterization of this state, in which various groups of agents are formed, each group with a distinct uniform opinion.

The paper is organized as follows. We define precisely the model in section 2 . We briefly recall in section 3 the results obtained in [32] concerning the comparison of the Deffuant model on static and adaptive networks, and in particular how the rewiring affects the transitions between consensus and polarized states. We focus in section 4 on the issue of convergence time, i.e. on how the parameters determine the time to reach a global or partial consensus. Finally, we consider in section 5 the detailed structure of 
the final state of the agents. We investigate in particular how the size of the various connected components, or groups of agents, is linked with their average number of connections.

\section{The model}

We consider the Deffuant model for the evolution of opinions, in which $N$ agents are endowed with a continuous opinion $o \in[0: 1][6,7,8,9,10]$. Starting from random values, the agents' opinions evolve through binary interactions according to the following rules: at each timestep $t$, two neighbouring agents are chosen at random. If their opinions are close enough, i.e., if $|o(i, t)-o(j, t)| \leq d$, where $d$ defines the tolerance range or threshold, they can communicate, and the interaction tends to bring them closer, according to the rule

$$
\begin{aligned}
& o(i, t+1)=o(i, t)+\mu(o(j, t)-o(i, t)) \\
& o(j, t+1)=o(j, t)-\mu(o(j, t)-o(i, t))
\end{aligned}
$$

where $\mu \in[0,1 / 2]$ is a convergence parameter. For the sake of simplicity and to avoid a too large number of parameters, we will consider the case of $\mu=1 / 2: i$ and $j$ adopt the same intermediate opinion after communication [7]. The tolerance parameter plays a crucial role in the ability of the population of agents to reach a global consensus or not. It is indeed intuitively clear that, for large tolerance values, agents can easily communicate and converge to a global consensus. On the contrary, small values of $d$ naturally lead to the final coexistence of several remaining opinions $[6,7]$.

For large populations, it may be more realistic to consider that the interactions between agents define a network with a finite average connectivity: each agent has a limited number of neighbours and can not a priori communicate with all the other agents. A typical example of such an interaction network structure is given by an uncorrelated random graph in which agents have $\bar{k}$ acquaintances on average, i.e. the initial network corresponds to an Erdős-Rényi network with average degree $\bar{k}$. While such a topology lacks many interesting features displayed by real social networks, such as degree heterogeneity or community structures, it is nevertheless interesting to first consider such a simple case as a reference frame. Moreover, we focus in this work on another important aspect of real networks: the fact that their topology may evolve on the same timescale as the agents' opinions. Agents can indeed break a connection or establish new ones, depending on the success of the corresponding relationship. The rules defining the evolution of the network topology can be modeled in many different ways. A possibility is to consider that links decay at a constant rate, independently from the agents' opinions [27]. In the case of opinion dynamics, we consider instead that only neighbouring agents with far apart opinions (i.e., $|o(i, t)-o(j, t)|>d$ ) may terminate their relationship [32]. In order to keep the average number of interactions constant, a new link is then introduced between one of the agents having lost a connection and another agent, chosen at random. The new link may of course break again if the newly 
connected agents have too-far-apart opinions. The rewiring process thus occurs as a random search for agents with close-enough opinions.

Even a simple rewiring process such as the one depicted above leads to the introduction of two new parameters. The first one, $w$, quantifies the relative frequencies of the two following processes: a local opinion convergence for agents whose opinions are within the tolerance range, and a rewiring process for agents whose opinions differ more. At each time step $t$, a node $i$ and one of its neighbors $j$ are chosen at random. With probability $w$, an attempt to break the connection between $i$ and $j$ is made: if $|o(i, t)-o(j, t)|>d$, the link $(i, j)$ is removed and a new link is created. With probability $1-w$ on the other hand, the opinions evolve according to (1) if they are within the tolerance range. The second parameter concerns the creation of a new link whenever a link $(i, j)$ has been removed: a new node $k$ is then chosen at random, and with probability $p \in[0: 1]$ a link $(i, k)$ is created, while with probability $1-p$ the new link instead connects $j$ and $k$. Since $j$ is chosen as a neighbour of a randomly chosen node $i$, it will have on average a larger degree. Larger $p$ therefore favors the removal of links from larger degree nodes, while small $p$ means that large degree nodes preferentially keep their links. We will see in section 5 how the parameter $p$ affects the final structure of the agents groups.

Both for static and adaptive networks, the system is initialized in a state where the agents have a random opinion between 0 and 1 and the network configuration is ErdösRényi. The averages were generated over 10 to 100 different networks, as specified in the captions of the figures. The dynamics stops when no update is anymore possible. If

$w>0$, this corresponds to a state in which no link connects nodes with different opinions. This can correspond either to a single connected network in which all agents share the same opinion, or to several disconnected clusters representing different opinions. For $w=0$ on the other hand, the final state is reached when neighboring agents either share the same opinion or differ of more than the tolerance $d$.

\section{Static versus adaptive}

In this section, we focus on the case $p=0$ and compare the results of the opinions evolution on a static and a dynamically adaptive network. Figure 1 displays for both cases the size of the largest $\left(\left\langle S_{\max }\right\rangle / N\right)$ and second largest $\left.\left(\left\langle S_{2}\right\rangle / N\right)\right)$ opinion clusters in the final state, where a cluster is defined as a connected group of agents sharing the same opinion. In both cases, at large tolerance $d$, a global consensus is achieved, with a single cluster containing all agents. A jump of $\left\langle S_{\max }\right\rangle / N$ from a value close to 1 to a value close to $1 / 2$ is observed at a critical value $d_{c 1}(w)$. Interestingly, and as hypothesized in [32], the structure of the largest cluster changes as $d$ decreases towards $d_{c 1}(w)$ : the average shortest path between two nodes increases as $d \rightarrow d_{c 1}(w)$ (data not shown), because the cluster typically acquires a community structure where only a few nodes or links keep the communities connected. Moreover, the critical value $d_{c 1}(w)$ increases with $w$ (see Fig. 1). If the rewiring is more frequent, it allows more easily to break the 
network in two pieces, since agents can more easily search for other agents with whom they can communicate, and break ties with the ones with too different opinions: the formation of different clusters is favored, and larger tolerance values are necessary to achieve consensus.
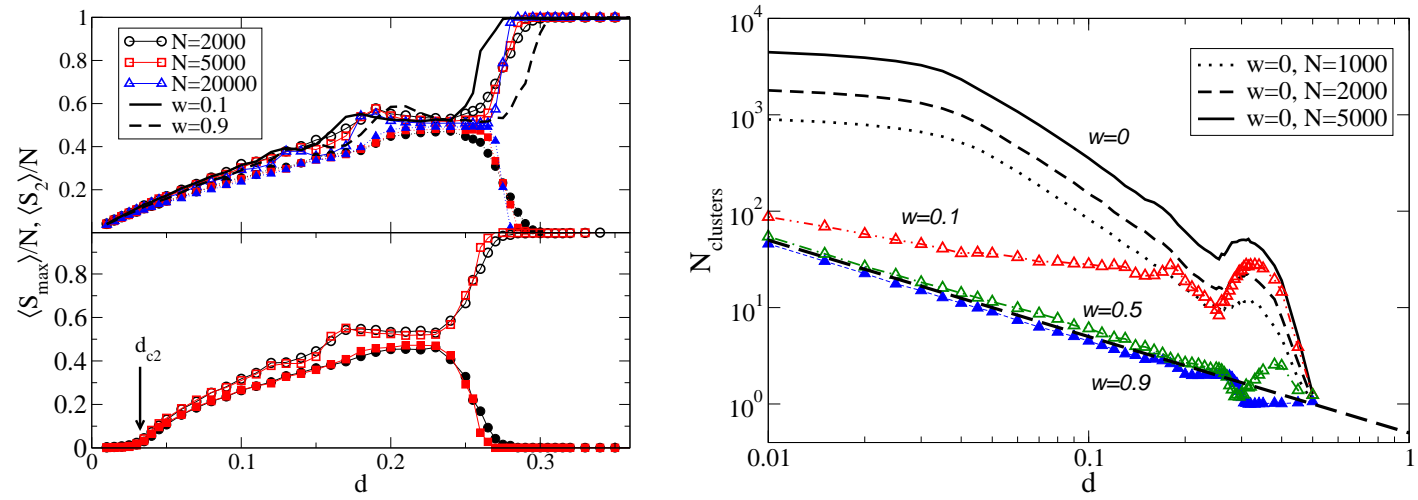

Figure 1. (Color online) Left figure: Size of the largest (empty symbols) and secondlargest (filled symbols) clusters in the final state, as a function of the tolerance value on a static Erdös-Rényi network with average degree $\bar{k}=10$ for different system sizes (bottom) and on adaptive networks with the same average degree and $w=0.5$. Continuous and dashed curves give the size of the largest cluster for $N=5000$ and $w=0.1$ and $w=0.9$, respectively: the consensus-to-polarized transition point is shifted to larger and larger tolerance values as the adaptivity of the network increases. Right figure: Number of clusters in the final state as a function of the tolerance of the agents for different system sizes on a static Erdös-Rényi network with $\bar{k}=10$, and on adaptive networks with the same average degree, $N=5000$, and different rewiring rates. The dashed line corresponds to the value $1 /(2 d)$, asymptotically valid at small tolerances in the mean-field case. Averages were generated over 100 different networks.

At $d<d_{c 1}$, an apparently polarized state is entered, with a first and second-largest clusters of similar extensive sizes, and apparently similar behavior for static and adaptive networks. A difference appears however at small tolerance values: for static networks, $\left\langle S_{\max }\right\rangle / N$ vanishes for $d<d_{c 2}$ in the thermodynamic limit. The final state of the system is then fragmented, with no cluster of extensive size. This polarized-fragmented transition is due to the finite connectivity of the agents and corresponds to a percolation phenomenon. An agent $i$ with $k$ connections and tolerance range $d$ will indeed have on average $2 d k$ neighbours with whom to communicate. For an average degree $\bar{k}<1 /(2 d)$, there will not be any percolating paths of agents with close enough opinions and only very small clusters of agreeing agents can be formed. In the case of adaptive networks on the other hand, the fragmented phase disappears as soon as the rewiring of the links is enabled. The size of the largest component decays smoothly but remains extensive as the tolerance decreases. Rewiring processes thus allow the small clusters, initially non-percolating, to create links between them and reach extensive sizes even below the polarized-to-fragmented transition appearing on static networks.

Further insight into the differences between static and adaptive networks is provided 
by the number of opinion clusters in the final state, $N_{\text {clusters }}$, shown in Fig. 1 . For $d_{c 2} \leq d \leq d_{c 1}$, an extensive number of clusters is indeed obtained in the static case, saturating at $\mathcal{O}(N)$ at $d_{c 2}$. The system presents therefore a "false"-polarized state, with a coexistence of macroscopic opinion clusters with an extensive number of finite size clusters. As $d$ decreases, more and more macroscopic clusters appear, as in meanfield [7], but there is also an extensive proliferation of finite size "microscopic" clusters. For adaptive networks, the number of clusters is much smaller, and decreases as $w$ increases. In fact, the precise investigation of the cluster size distribution reveals that the density of nodes in non-extensive clusters vanishes in the thermodynamic limit [32]. The polarized phase on adaptive networks differs therefore strongly from the one on static networks: thanks to the possibility of link rewiring, agents who would remain isolated (or in very small groups) on a static network may manage to find agents with whom to communicate and thus enter a macroscopic cluster. Without rewiring on the other hand, a macroscopic number of agents remain in fragmented components which coexist with few macroscopic clusters.

\section{Convergence time}

On static networks, the time to converge to the final state of the system, $t_{c o n v}$, is determined by the topological properties of the opinion clusters. In turn, the behavior of this topology is mostly determined by the distance of the tolerance of the agents from that of the polarized-to-fragmented transition, $d_{c 2}$ (see Fig. 2): For $d_{c 2}<d, t_{c o n v}$ grows linearly with the system size and increases as $d$ decreases: the clusters formed by agents who can communicate become more and more tree-like, which slows down the convergence to a common opinion. As $d \rightarrow d_{c 2}, t_{c o n v}$ diverges as a signature of the phase transition. For $d<d_{c 2}$, the clusters of agents with close enough opinions become small and the convergence time decreases as $d$ decreases.

On adaptive networks, two scenarios are possible: on the one hand, if the network evolution is slow compared to the timescale of opinion formation, $t_{c o n v}$ is mostly determined by the characteristic time of topological cluster formation, $t_{l} \ddagger$. The scaling of $t_{l}$ and therefore $t_{c o n v}$ can be estimated by considering a typical opinion cluster: its size is proportional to the tolerance range of the agents, $2 d$; the number of its links which need to be rewired is proportional to the total number of links $(\propto \bar{k} N)$, and to the amount of opinions outside of the tolerance range $(\propto(1-2 d))$; the probability to rewire a link towards an agent with a close enough opinion is moreover $\propto d$ and the time between two link updates is $\propto 1 / w$ so that

$$
t_{l} \propto \bar{k}(1-2 d) /(w d) .
$$

Figure 2B) shows the rescaled convergence time $w t_{\text {conv }} / \bar{k}$ as a function of $d$ for different parameter values, in good agreement with Eq. (2). If the network evolution is fast, on

$\ddagger$ It is important to note that, even in this limit, the network topology cannot be considered static and adaptivity plays an important role in the early time evolution of the system too, as shown in [32]. 

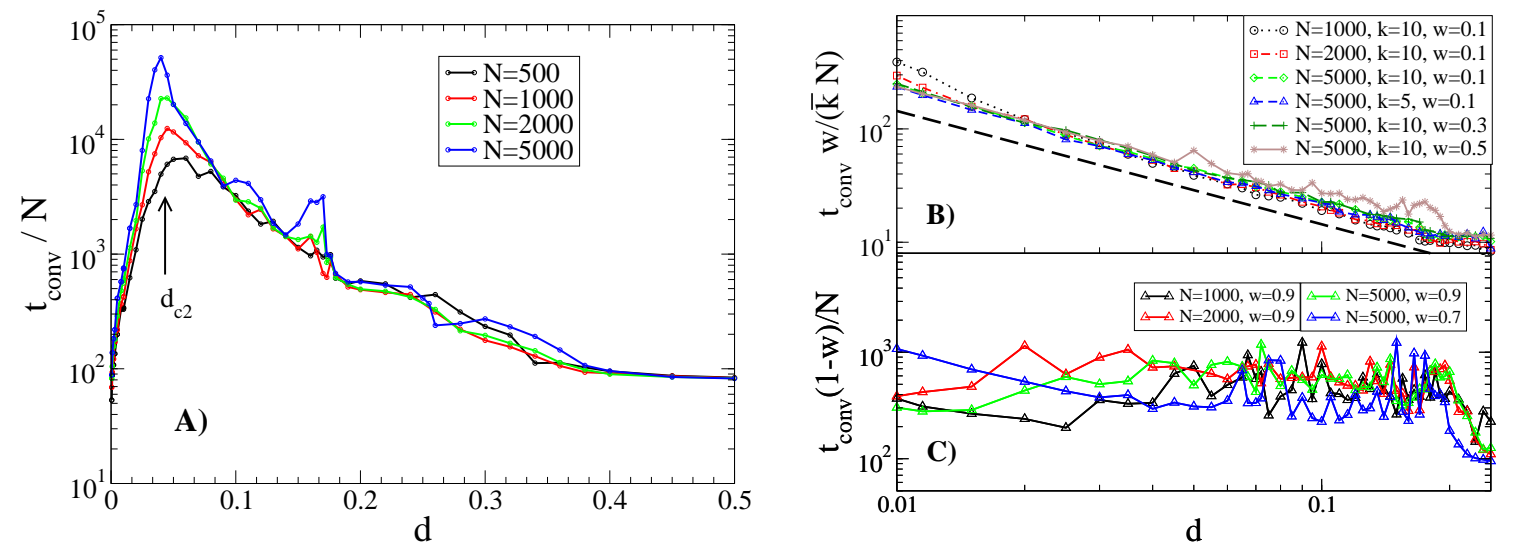

Figure 2. (Color online) A) convergence time, measured in the number of simulation steps, on static networks for different system sizes as a function of the tolerance, $d$, when $\bar{k}=10$ (The data points were generated averaging over 10 to 100 realizations). B) rescaled convergence time on adaptive networks for small rewiring rates as a function of $d$ for different system sizes, average degrees, and rewiring rates (Data points averaged over 30 realizations.). The dashed line is proportional to $1 / d$. C) Rescaled convergence time on adaptive networks as a function of $d$ for different system sizes and rewiring rates $(\bar{k}=10)$ averaged over 100 realizations.

the other hand, it is possible for an agent to rewire most links towards agents with close opinions in a short time. This scenario takes place when $t_{l}$ is less than or comparable to $1 /(1-w)$, the average time between two fruitful discussions. In this case, the convergence time is expected to scale simply as $1 /(1-w)$, as indeed shown in Fig. $2 \mathrm{C})$.

\section{Group structure}

Once the population of agents has reached its final state, an interesting question concerns the structural differences between the various opinion groups that have been formed. The most basic property one can investigate is the average degree of an agent. It turns out that the average degree of an agent inside a group is strongly correlated with the size of the group. On static networks, the average degree of a cluster is a linear function of its size (left plot of Fig. 3), which can be explained as follows: for a cluster of size $S$, the probability for a node to have a link pointing towards this cluster is simply $S / N$, and a node of degree $k$ will have on average $k S / N$ links pointing towards other nodes in the cluster. The average "in-degree" of the nodes in a cluster of size $S$ is therefore $S \bar{k} / N$ (assuming that there is no correlation between the degree of a node in the network and the cluster to which it finally belongs) §.

On adaptive networks on the other hand, the linear relationship is no longer valid, as shown in Fig. 3. At small tolerance values, many clusters are obtained, with very $\S$ Moreover, the average degree cannot be less than two, except for starlike clusters where the average degree can be between 1 and 2, which explains the flattening of the curves at small cluster sizes. 
different sizes. A power-law-like relationship appears between the clusters' size and degree. The behaviour depends on $p$, the parameter of the rewiring rule: a sublinear relationship holds for small $p$ values while a superlinear behaviour appears for $p$ close to 1. Since these cases correspond to the situations when the development of the opinion clusters takes place at a much shorter timescale than that of the topological clusters [32], an analytical treatment of the problem is possible, investigating the diffusion of the links between the clusters of constant opinions at a mean-field level [33]. For large tolerance values, the cluster's average degree becomes less correlated with its size (right graph of Fig. 3): this is due to the fact that the clusters correspond to large fractions of the original network and their average degree saturates at $\bar{k}$.
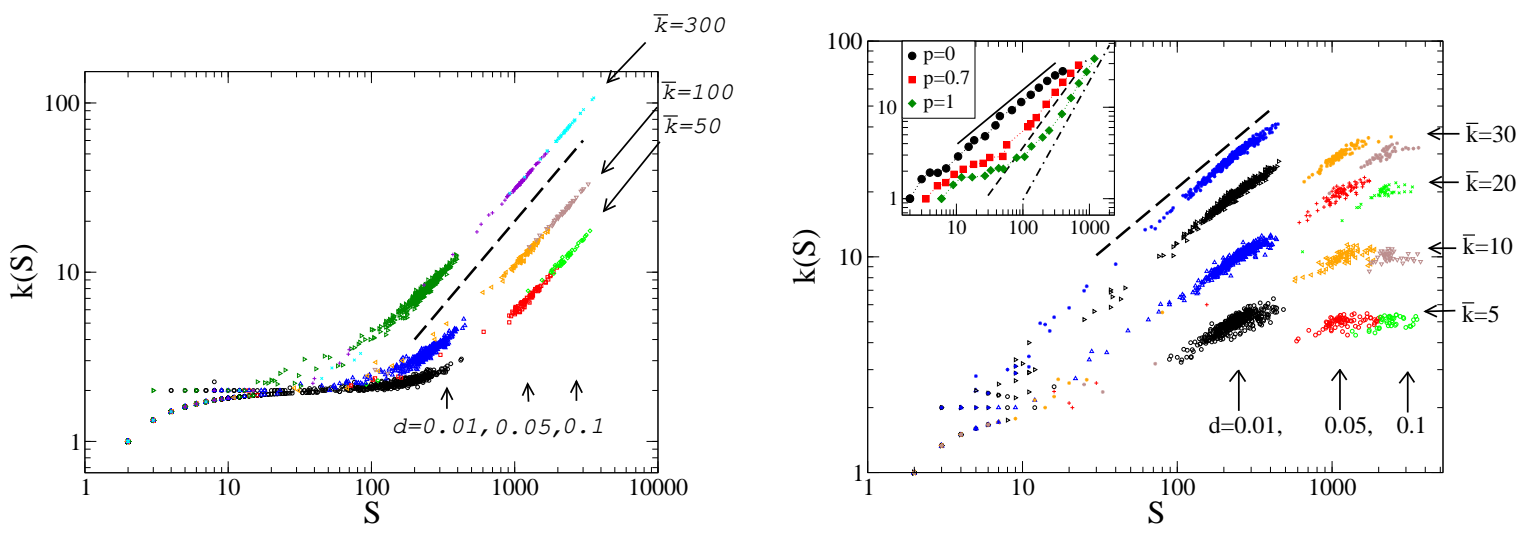

Figure 3. (Color online) Left figure: average degree of the agents sharing the same opinion as a function of the size of the uni-opinion groups, $S$, on static networks. The dashed line corresponds to a linear behaviour. Right figure: same in the case of adaptive networks when $w=0.5$ and $p=0$ for different average degrees and tolerance values. The dashed line shows a power-law $S^{0.6}$. Inset: the binned average of the same measurement for $\bar{k}=20, d=0.01, w=0.5$, and different values of $p$. The lines correspond to the power laws $S^{0.6}, S$, and $S^{1.3}$. In both figures, $N=10^{4}$ and data points were collected from 10 realizations for each set of parameter values.

\section{Conclusions}

In this work we have investigated how adaptive network topology can influence the final state and structure of the Deffuant model compared to its behavior on static ErdösRényi networks. While on static networks the model exhibits three different phases, the fragmented phase, present at very small tolerance values and characterised by the lack of extensive-size clusters, disappears on adaptive networks since rewiring allows small groups to connect to each other and grow to macroscopic sizes for all tolerance values. The consensus-to-fragmented transition is shifted to larger tolerance values on adaptive networks since rewiring promotes the division of agents with too different opinions. We have found that the convergence time of the system on static networks is determined by the distance of the tolerance of the agents from that at the polarized-to-fragmented 
transition, $d_{c 2}$. On adaptive networks instead, the convergence time is set either by the time it takes to successfully rewire the links between disagreeing agents or simply by the frequency of opinion updates, depending on which of these timescales is larger than the other. Finally, probing the local structure of the groups by measuring the average degree of them reveals nontrivial correlations between the size of a group and its average degree: The average degree is either a sub or superlinear function of the size, determined by the parameter of the rewiring rule, $p$. Adaptive dynamics moreover display robust features with respect to small changes in the opinion-update rule contrarily to what happens on static networks, on which for example the consensus-to-polarized transition disappears when the tolerance threshold is not sharp anymore [32].

\section{Acknowledgments}

The authors are partially supported by the EU under contract 001907 (DELIS).

\section{References}

[1] Marro J, Garrido P L and Muñoz M A (Eds.), AIP Conf. Proc. 779, Granada, Spain (2005); Weidlich W 2000 Sociodynamics; A Systematic Approach to Mathematical Modelling in Social Sciences, (Harwood Academic Publishers); Stauffer D, Moss de Oliveira S M, Murilo Castro de Oliveira P and Simoes de Sá Martins J 2006 Biology, Sociology, Geology by Computational Physicists (Elsevier Science)

[2] Galam S, Gefen Y and Shapir Y, Math. J. Soc. 9, 1 (1982).

[3] Krapivsky P-L 1992 Phys. Rev. A 45, 1067

[4] Krapivsky P-L and Redner S 2003 Phys. Rev. Lett. 90, 238701

[5] Axelrod R 1997 J. of Conflict Resolut. 41, 203

[6] Deffuant G, Neau D, Amblard F and Weisbuch G 2000 Advances in Complex Systems 3, 87

[7] Ben-Naim E, Krapivsky P-L and Redner S 2004 Physica D 183, 190

[8] Stauffer D and Meyer-Ortmanns H 2003 Int. J. Mod. Phys. C 15, 241

[9] Ben-Naim E 2005 Europhys. Lett. 69, 671

[10] Amblard F and Deffuant G 2004 Physica A 343, 725

[11] Steels L 1996 Artificial Life Journal 2, 319

[12] Baronchelli A, Felici M, Loreto V, Caglioti E and Steels L 2006 J. Stat. Mech. P06014

[13] Dall'Asta L, Baronchelli A, Barrat A and Loreto V 2006 Phys. Rev. E 74, 036105

[14] Granovetter M 1973 American Journal of Sociology 78, 1360

[15] Wasserman A and Faust K 1994 Social Network Analysis: Methods and applications (Cambridge University Press, Cambridge)

[16] Albert R and Barabási A-L 2002 Rev. Mod. Phys. 74, 47

[17] Dorogovtsev S N and Mendes J F F 2002 Adv. Phys. 51, 1079

[18] Dorogovtsev S N and Mendes J F F 2003 Evolution of networks: From biological nets to the Internet and $W W W$ (Oxford University Press, Oxford)

[19] Newman M E J 2003 SIAM Review 45, 167

[20] Pastor-Satorras R and Vespignani A 2004 Evolution and structure of the Internet: A statistical physics approach (Cambridge University Press, Cambridge)

[21] González M C, Lind P G and Herrmann H J 2006 Eur. Phys. J. B 49, 371-376

[22] Castellano C, Loreto V, Barrat A, Cecconi F and Parisi D 2005 Phys. Rev. E 71, 066107

[23] Sood V and S. Redner S 2005 Phys. Rev. Lett. 94, 178701

[24] Suchecki K, Eguíluz V M and San Miguel M 2005 Europhys. Lett. 69, 228 
[25] Suchecki K, Eguíluz V M and San Miguel M 2005 Phys. Rev. E 72, 036132

[26] Zimmermann M G, Eguíluz V M and San Miguel M 2004 Phys. Rev. E 69, 65102

[27] Ehrhardt G, Marsili M and Vega-Redondo F 2006 International Journal of Game Theory 34, 383

[28] Gil S and Zanette D H 2006 Physica D 224, 156

[29] Holme P and Newman M E J 2006 Phys. Rev. E 74, 056108

[30] Garlaschelli D, Capocci A and Caldarelli G 2007 Nature Physics 3, 813

[31] Stauffer D, Hohnisch M and Pittnauer S 2006 Physica A 370, 734

[32] Kozma B and Barrat A 2008 Phys. Rev. E 77, 016102

[33] Gautreau A and Kozma B, in preparation. 